\title{
A patient-specific model of total knee arthroplasty to estimate patellar strain: A case study
}

\author{
Adeliya Latypova ${ }^{\mathrm{a}}$, Arash Arami ${ }^{\mathrm{b}}$, Fabio Becce ${ }^{\mathrm{c}}$, Brigitte Jolles-Haeberli ${ }^{\mathrm{d}}$, Kamiar Aminian ${ }^{\mathrm{b}}$, \\ Dominique P. Pioletti ${ }^{\mathrm{a}}$, Alexandre Terrier ${ }^{\mathrm{a}, *}$ \\ a Ecole Polytechnique Fédérale de Lausanne (EPFL), Laboratory of Biomechanical Orthopedics, Lausanne, Switzerland \\ ${ }^{\mathrm{b}}$ Ecole Polytechnique Fédérale de Lausanne (EPFL), Laboratory of Movement Analysis and Measurement, Lausanne, Switzerland \\ ${ }^{c}$ Lausanne University Hospital (CHUV), Department of Diagnostic and Interventional Radiology, Lausanne, Switzerland \\ d Vaudois Lausanne University Hospital (CHUV) and University of Lausanne, Lausanne, Switzerland
}

\section{A R T I C L E I N F O}

\section{Article history:}

Received 21 May 2015

Accepted 19 November 2015

\section{Keywords:}

Knee

Arthroplasty

Patella

Strain

Kinematics

Finite elements

\begin{abstract}
A B S T R A C T
Background: Inappropriate patellar cut during total knee arthroplasty can lead to patellar complications due to increased bone strain. In this study, we evaluated patellar bone strain of a patient who had a deeper patellar cut than the recommended.

Methods: A patient-specific model based on patient preoperative data was created. The model was decoupled into two levels: knee and patella. The knee model predicted kinematics and forces on the patella during squat movement. The patella model used these values to predict bone strain after total knee arthroplasty. Mechanical properties of the patellar bone were identified with micro-finite element modeling testing of cadaveric samples. The model was validated with a robotic knee simulator and postoperative X-rays. For this patient, we compared the deeper patellar cut depth to the recommended one, and evaluated patellar bone volume with octahedral shear strain above $1 \%$.

Findings: Model predictions were consistent with experimental measurements of the robotic knee simulator and postoperative X-rays. Compared to the recommended cut, the deeper cut increased the critical strain bone volume, but by less than $3 \%$ of total patellar volume.

Interpretation: We thus conclude that the predicted increase in patellar strain should be within an acceptable range, since this patient had no complaints 8 months after surgery. This validated patient-specific model will later be used to address other questions on groups of patients, to eventually improve surgical planning and outcome of total knee arthroplasty.
\end{abstract}

(c) 2015 Elsevier Ltd. All rights reserved.

\section{Introduction}

Despite relatively high success rate of total knee arthroplasty (TKA), $11 \%$ to $19 \%$ of primary TKA patients are not satisfied with the surgical outcome and around $6 \%$ of patients go for a revision surgery due to postoperative complications (Bourne et al., 2010; Bozic et al., 2010; Pabinger et al., 2013; Von Keudell et al., 2014). Among those, up to 24\% of the revision surgeries can be caused by complications related to the patella: osteonecrosis, patellar fracture, implant failure, polyethylene wear, extensor mechanism rupture, and anterior knee pain (AKP) (Bozic et al., 2010; Schindler, 2012; Sundberg et al., 2014).

In vivo (Carpenter et al., 2009; Sharma et al., 2012), in vitro (Lie et al., 2005; Matsuda et al., 1997; Merican et al., 2014) and in silico (Amirouche et al., 2013; Fitzpatrick et al., 2011; Takahashi et al., 2012) studies have shown that TKA leads to significant changes in patellar

* Corresponding author at: Laboratory of Biomechanical Orthopedics, Ecole Polytechnique Fédérale de Lausanne, Station 19, 1015 Lausanne, Switzerland.

E-mail address: alexandre.terrier@epfl.ch (A. Terrier). kinematics, forces acting on the patella, and resulting bone strain in comparison with the intact knee. One of the possible causes of these changes can be related to a surgical technique influencing the positioning of the prosthetic components, their rotation around the joint axes, and the amount of bone cut. Even though surgical techniques have been improved during the last decades, and navigational systems as well as patient-specific cutting guides make the surgery more controllable (Franceschi and Sbihi, 2014), patellar resurfacing still remains the less controllable part of the procedure. Small size of the patellar bone, surrounding soft tissues and osteophytes, difficulty of finding anatomical landmarks, and thickness of the cutting jigs can easily lead to deeper bone cut or unwanted resection angles (Anglin et al., 2009).

Several numerical and in vitro studies showed that reduction of patellar thickness can lead to increase of patellar bone strain (Amirouche et al., 2013; Fitzpatrick et al., 2013; Lie et al., 2005), thus increasing risk of the patellar fracture and possibly contributing to AKP symptoms (Draper et al., 2012; Ho et al., 2014). However, these studies were conducted on cadaveric knees. To the best of our knowledge, no studies tried to estimate patellar bone strain and effect of bone resection on 
real TKA patients. The estimation of the strain in TKA patients could help to evaluate strain ranges that should be kept after surgery in order to avoid complications. Such knowledge in combination with a patientspecific numerical model would help to improve patellar resection technique during surgery planning. Moreover, all numerical models predicting patellar bone strain assign elastic properties measured on other bones than the patella (Fitzpatrick et al., 2011; Ho et al., 2014; Takahashi et al., 2012) due to the limited number of studies focused on the measuring of patellar mechanical properties.

Hypothesizing that an inappropriate patellar cut increases the risk of postoperative patellar complications, and that numerical model could help to estimate this risk through prediction of patellar bone strain, in the present study we investigated the retrospective case of a TKA patient who had a resection of the patellar bone that was deeper than usually recommended. The first objective was to develop and validate a patient-specific TKA model replicating squat movement. This model should be able to predict postoperative patellar strain using patient preoperative data, such as CT scan, height, weight, implant design, and preoperative planning. Our second objective was to use this model to analyze the influence of the patellar cut depth on patellar strain.

\section{Methods}

A patient-specific TKA numerical model was developed from preoperative data of a TKA patient. A loaded squat movement was simulated. The TKA model was decoupled into two levels: knee and patella. The knee model predicted kinematics and forces on the patella. The patella model used these values to predict bone strain. For this patient, we compared the effect of the patellar cut depth on patellar kinematics and strain.

\subsection{TKA patient}

The TKA patient chosen for this study was a 78-year-old female $(156 \mathrm{~cm}, 68 \mathrm{~kg})$. A specific TKA preoperative CT protocol was designed to obtain the 3D volume-rendered reconstructions of the knee joint and thigh muscles, and to estimate the centers of the hip and ankle joints (Fig. 1a). CT data were acquired on a multidetector CT scanner (Discovery CT750 HD, GE Healthcare, Milwaukee, USA). The standard
CT protocol was extended by adding a set of eighteen 1.25 -mm-thick slices evenly distributed along thigh muscles from the acetabular roof to femoral condyles (Narici et al., 1992). The patient received a cemented ultra-congruent posterior-stabilized mobile-bearing knee prosthesis (F.I.R.S.T.; Symbios, Yverdon-les-Bain, Switzerland) with a three-peg modified dome patellar component. Patient-specific cutting guides were used to insert the femoral and tibial components (Franceschi and Sbihi, 2014). The patient was followed-up 8 months after surgery. The postoperative X-rays revealed a $12 \mathrm{~mm}$ patellar cut depth instead of the recommended $9 \mathrm{~mm}$ corresponding to the thickness of the patellar component (Fig. 2).

\subsection{Knee model}

The knee model included the femur, tibia, patella, quadriceps and hamstring (Fig. 1c). We considered the four quadriceps muscles: rectus femoris (RF), vastus intermedius (VI), vastus lateralis (VL), vastus medialis (VM); and the three hamstring muscles: biceps femoris (BF), semitendinosus (ST), and semimembranosus (SM). Bone and muscle geometry was reconstructed by segmentation of CT images (Amira; FEI Visualization Sciences Group, Burlington, USA) (Fig. 1b). In case of muscle reconstruction, the segmentation was interpolated between eighteen slices. The prosthesis was positioned into the model according to preoperative planning, and verified with postoperative X-ray. Patellar cut was estimated from postoperative X-ray (lateral and sunrise view).

We implemented the knee model in Abaqus (Simulia, Providence, USA). Bones and metallic components were rigid. Polyethylene tibial insert and patellar button were linear elastic $(\mathrm{E}=572 \mathrm{MPa}, v=0.45)$ (Fitzpatrick et al., 2011). Patellar tendon was modeled by two nonlinear springs (Staubli et al., 1996, preconditioned state). Medial and lateral patellofemoral ligaments were modeled by two linear fibers each with 10\% pre-strain (Atkinson et al., 2000; Marra et al., 2015; Merican et al., 2009). Quadriceps tendons were linear fibers ( $E=1.2 \mathrm{GPa}$, Cross Sectional Area $=62.5 \mathrm{~mm}^{2}$ ) (Voigt et al., 1995) and could wrap around femoral surface at deep angles of flexion. The seven muscles were represented by seven pair of nodes defining muscle lines of action. Directions of four quadriceps muscles in frontal plane were taken from literature (Sakai et al., 1996, anatomical model), while to define position in sagittal plane the lines were directed through the muscle origins on

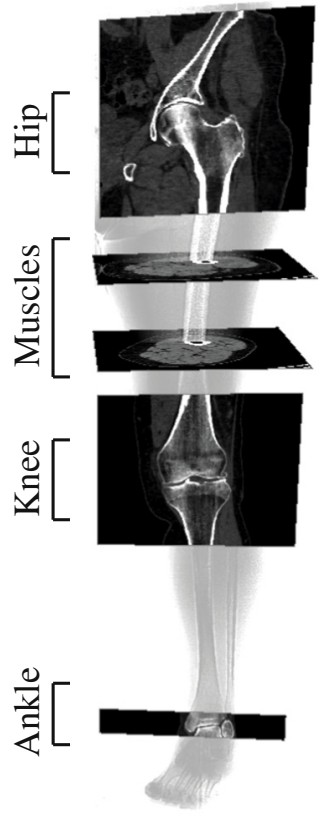

(a)
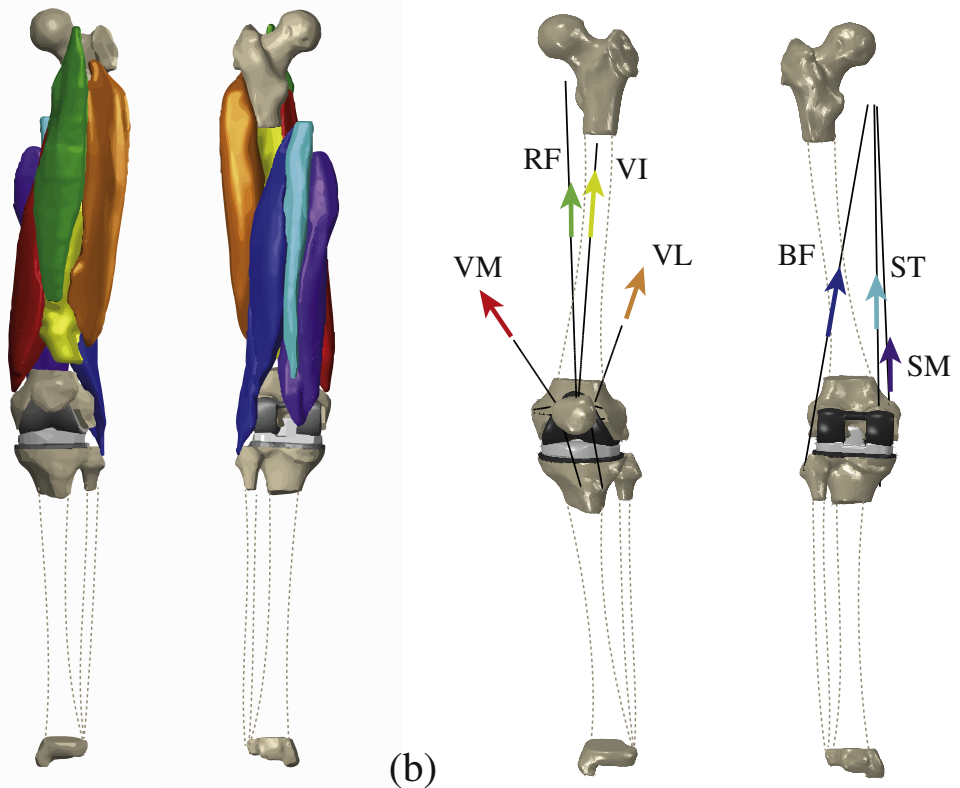

(c)

Fig. 1. Patient preoperative CT dataset: hip, knee, and ankle joints, and thigh muscles (a); anterior and posterior views of 3D volume-rendered reconstruction of bones, muscles, and prosthesis (b); and anterior and posterior views of the knee joint model (c). 
Received cut

Recommended cut

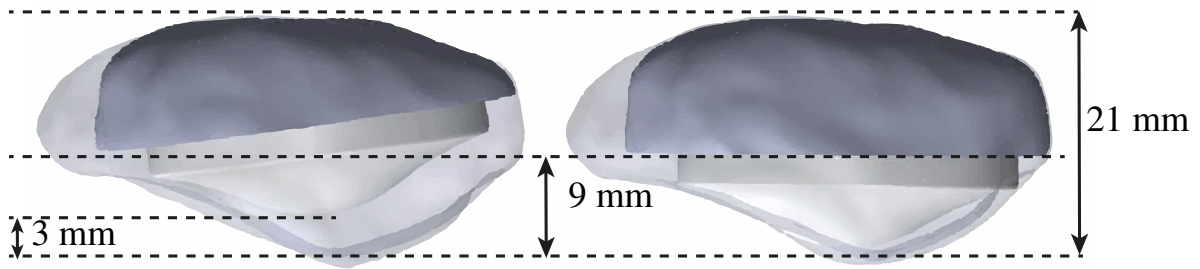

Fig. 2. Patient received (left) and recommended (right) patellar cut in comparison with intact patellar geometry.

the patella tangent to the reconstructed muscle volumes. Directions of hamstring muscles were taken as lines connecting approximate insertions and origins of the muscles on the tibia and pelvis. The VI was chosen as a reference muscle. The knee flexion was controlled by applied half body weight and VI lengthening. At each increment of the nonlinear implicit solver, the length of other six muscles and forces in all muscles were recalculated to keep moment equilibrium of the system. Forces were controlled by a user-defined element (UEL) to guarantee predefined force ratios (Terrier et al., 2007). These muscle force ratios were defined as the product of their physiological cross-sectional area (PCSA) and electromyography (EMG), normalized by the product of PCSA and EMG of VI (Table 1). PCSA was calculated by dividing muscle volume by fiber length. Fiber length $\left(L_{F}\right)$ was estimated through muscle length $\left(\mathrm{L}_{\mathrm{M}}\right)$ and known $\mathrm{L}_{\mathrm{F}} / \mathrm{L}_{\mathrm{M}}$ ratios (Ward et al., 2009). EMG was estimated from measurements on healthy volunteers (Dionisio et al., 2008; Isear et al., 1997; Yavuz et al., 2015). Since EMG signals of these muscles were nearly proportional during squat, their force ratios were assumed constant. EMG of VI and RF were assumed to be equal. We simulated so called Oxford squat: the half of the BW was applied on the hip along the vertical axis passing through the hip and ankle centers during all movement. The hip could translate only along this axis, as well as flex/extend in the sagittal plane and adduct/abduct in the frontal plane. The ankle had the same rotational degrees of freedom, but was fixed in translations.

We modeled the patellofemoral and tibiofemoral contact, contact between the quadriceps tendons, patellofemoral ligaments and femoral component as frictionless hard contact. The deformable polyethylene components were meshed with quadratic tetrahedral elements, while the femoral rigid surface with quadrilateral elements. The quadriceps tendons and patellar ligament were meshed with truss and tensiononly connector elements respectively. The patellofemoral ligaments included truss elements used to model contact with femoral component and tension-only connector elements to simulate pre-strain.

The predicted patellar kinematics was described with a threecylindrical open chain system (Grood and Suntay, 1983). Patellar and femoral coordinate systems were chosen according to prostheses designs (Sharma et al., 2012). For the patella, the anterior-posterior (A-P) axis coincided with the axis of the conical articular dome, medial-lateral (M-L) axis was set as bisector of medial and lateral pegs lying on the anterior flat face of the patellar component. The superior-

\section{Table 1}

Estimated muscle PSCA through muscle volume $(V)$, muscle length $\left(L_{\mathrm{M}}\right)$, and normalized fiber length $\left(L_{\mathrm{F}}\right)$ estimated through muscle length, normalized EMG, and final muscle ratios.

\begin{tabular}{lllllll}
\hline Muscle & $V\left(\mathrm{~cm}^{3}\right)$ & $L_{\mathrm{M}}(\mathrm{cm})$ & $L_{\mathrm{F}}(\mu \mathrm{m})$ & $\begin{array}{l}\text { PSCA } \\
\left(\mathrm{cm}^{2}\right)\end{array}$ & $\begin{array}{l}\text { EMG } \\
(\%)\end{array}$ & $\begin{array}{l}\text { Final } \\
\text { ratios }\end{array}$ \\
\hline RF & 118.6 & 27.2 & 63.9 & 18.6 & 38 & 0.60 \\
VL & 367.5 & 28.7 & 98.1 & 37.4 & 46 & 1.46 \\
VM & 276.3 & 30.3 & 80.8 & 34.2 & 48 & 1.41 \\
VI & 252.0 & 27.2 & 81.6 & 30.9 & 38 & $\mathbf{1 . 0 0}$ \\
BF $(S+L)$ & $79.7+171.8$ & $21.7+27.4$ & $87.1+88.4$ & $9.2+19.4$ & 7 & 0.17 \\
ST & 116.9 & 25.7 & 156.6 & 7.5 & 6 & 0.04 \\
SM & 220.7 & 26.9 & 67 & 32.9 & 6 & 0.17 \\
\hline
\end{tabular}

inferior (S-I) axis was a cross product of A-P and M-L axes. For the femur, the M-L axis was set as line between centers of the main femoral condyles spherical parts. The S-I axis was set as line between midpoint of centers of the main femoral condyles spherical parts and femoral head center. The A-P axis was a cross product of M-L and S-I axes. Translations were $\mathrm{M}-\mathrm{L}, \mathrm{A}-\mathrm{P}$ and $\mathrm{S}-\mathrm{I}$, where medial, anterior and superior translations were positives. Rotations were flexion-extension, M-L spin and M-L tilt, where flexion, lateral spin, and lateral tilt were positives.

Patella kinematics and quadriceps force predictions were validated with a robotic knee simulator (Appendix A). The model was adapted to replicate an existing robotic knee simulator (Arami et al., 2015). We compared the model predictions with the simulator measurements during a quasi-static loaded squat movement, from $10^{\circ}$ to $60^{\circ}$ of knee flexion. Since the tendon of the model did not replicate perfectly the geometry of the tendon of the simulator (thickness, not flat attachment to the patellar surface), we evaluated the sensitivity of the patellar tendon insertion on the kinematics and force predictions. The difference between predictions and measurements was estimated with root mean square error (RMSE). In addition, we verified the quadriceps force prediction by comparison with a 2D pulley-like algebraic model.

The patient-specific prediction of patella position was compared to the postoperative lateral view (full extension, weight bearing) and sunrise view (non-weight bearing, $30^{\circ}$ of knee flexion) X-rays at 3 months. To simulate non-weight bearing $30^{\circ}$ of flexion, quadriceps force of $35 \mathrm{~N}$ was distributed among its heads according to muscle CSA (Anglin et al., 2008).

\subsection{Patella model}

The patella model consisted of the patellar bone, polyethylene component, cement layer, and femoral component. Initial position of all elements coincided with their initial position in the knee model. Patella and femoral component were then moved to the position predicted by the knee model. The femur was then completely fixed, while the patella was only constrained in the superior-inferior direction. Patella forces (quadriceps, patellar tendon, patellofemoral ligaments) predicted by the knee model were distributed along the anterior patellar surface. Cement was linear elastic ( $E=3.4 \mathrm{GPa}, \nu=0.3$ ) (Fitzpatrick et al., 2011). Bone and cement were meshed with linear tetrahedral elements, while PE with quadratic tetrahedral elements. We considered the octahedral shear strain invariant since it can be associated with bone damage and remodeling (Isaksson et al., 2007; Kettenberger et al., 2015).

Patellar bone was linear elastic, but elasticity tensor depended on bone volume fraction $(\rho)$ (Zysset, 2003):

$E=E_{0} \rho^{k}, v=v_{0}$

We identified the three constants $\left(E_{0}, \nu_{0}, k\right)$ using a regression analysis with micro-finite element ( $\mu \mathrm{FE}$ ) testing (Gross et al., 2013) on 20 fresh-frozen cadaveric patellae ( 8 female, 12 male, mean age $67 \pm$ 17 years). We virtually extracted 10 cubes $(5.3 \mathrm{~mm})$ from each scanned (Skyscan 1076; Bruker microCT, Kontich, Belgium) patella. All cubes were converted into $\mu \mathrm{FE}$ models with isotropic hex elements with $E=12 \mathrm{GPa}, v=0.3$ for the bone tissue (Wolfram et al., 2010) (Fig. 3). 

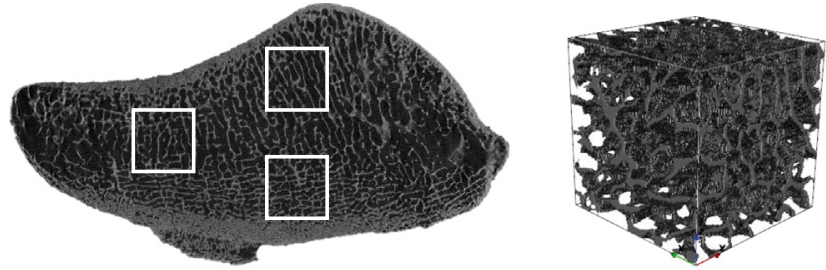

Fig. 3. Example of the patellar $\mu \mathrm{CT}$ scan with position of the cubes (left) and a segmented cubic sample (right).

We simulated three compressions and three shears for each cube. Preand post-processing were performed with MEDTOOL (www.dr-pahr. at), and $\mu \mathrm{FE}$ solver was ParFE (parfe.sourceforge.net).

Since the patient CT scan does not provide bone volume fraction $(\rho)$, but bone mineral density (BMD), we correlated $\rho$ from $\mu \mathrm{CT}$ to BMD from CT with the same cadaveric patellae:

$\rho=1.0614 B M D-0.0573$

BMD was obtained from Hounsfield units and calibration phantom (Mindways Software, Austin, USA)

\subsection{Sensitivity analysis}

We evaluated the influence of the patient body weight (BW \pm $100 \mathrm{~N})$, the distribution of the VM (VM ratio $\pm 25 \%$ ) and VL forces (VL ratio $\pm 34 \%), \mathrm{VM}$ and $\mathrm{VL}$ force direction in frontal plane $\left(+5^{\circ}\right.$ laterally/medially) on the knee and patellar models predictions. For the patellar model, we first evaluated sensitivity of bone strain predictions on the finite element mesh. We compared an average edge size of $1.5 \mathrm{~mm}$, $1.0 \mathrm{~mm}$ and $0.8 \mathrm{~mm}$. The strain verification was performed at $60^{\circ}$ of knee flexion.

\subsection{Patellar cut}

For this patient, we compared the performed patellar cut depth $(12 \mathrm{~mm})$ to the recommended one $(9 \mathrm{~mm})$, and evaluated its effect on bone strain. We compared bone volume above $1 \%$ and $2 \%$ of octahedral shear strain as these strain levels are often associated with bone yield (Carter et al., 1981; Lammentausta et al., 2006; Morgan and Keaveny, 2001). Difference in strain distribution within the bone volume in two cases was estimated using a two-sample KolmogorovSmirnov test with $95 \%$ confidence interval.

\section{Results}

\subsection{Patellar kinematics and forces}

During knee flexion, patellar flexion angle grew almost linearly from $9^{\circ}$ to $64^{\circ}$ (Fig. 4). Patellar tilt decreased from $5^{\circ}$ to $1^{\circ}$ during first $60^{\circ}$ of knee flexion, and remained nearly constant thereafter. Patellar spin remained nearly constant during knee flexion. Quadriceps and patellar tendon forces increased almost linearly during knee flexion, reaching 5 and $4.2 \mathrm{BW}$ respectively. The patellofemoral force reached $6 \mathrm{BW}$ at $90^{\circ}$ of knee flexion.

Compared to the recommended patellar resection, the deeper received cut induced slightly higher patellar flexion $\left(2^{\circ}\right)$, during the first $60^{\circ}$ of knee flexion. The difference slowly diminished to $0.5^{\circ}$ by the end of movement (Fig. 4). The patellar spin decreased by $3^{\circ}$, and the tilt was not affected. Lateral shift was $1 \mathrm{~mm}$ higher at full extension, and nearly equal after $30^{\circ}$ of knee flexion. PF force decreased by $0.2 \mathrm{BW}$, while PT force increased by less than $0.3 \mathrm{BW}$. Quadriceps force decreased after $75^{\circ}$ of flexion by 0.15 BW.

For the validation with the robotic simulator, the RMSE between predicted and measured patellar M-L, A-P and S-I translations were $0.5,0.3$ and $1.1 \mathrm{~mm}$, respectively. The RMSEs between predicted and measured patellar flexion, spin and tilt were $0.6^{\circ}, 0.2^{\circ}$ and $2.3^{\circ}$, respectively. The RMSE of quadriceps force between the model and robotic simulator was of $120 \mathrm{~N}$ (mean relative error of $10 \%$ ). The errors of the experimental measurements were less than $0.8 \mathrm{~mm}$ for translations, $2.4^{\circ}$ for rotations, and $32 \mathrm{~N}$ for the quadriceps force. The sensitivity analysis of the patellar tendon insertion position on the patellar kinematics revealed a negligible effect below $40^{\circ}$ of knee flexion. Above this flexion angle, the maximal variations were $1.5 \mathrm{~mm}$ for translations and $1.1^{\circ}$ for rotations. However, quadriceps force was sensitive to tendon position and the average relative difference between two extreme cases was $6 \%$. The verification of the numerical model with the algebraic one showed a mean relative difference of $0.5 \%$ between the predicted and theoretical quadriceps force. For the patient, the predicted patellar position was similar to the postoperative X-ray (Fig. 5).

Variation of the BW by $15 \%(100 \mathrm{~N})$ did not lead to noticeable changes in patellar kinematics, while forces were proportional to the changes in BW. Variation of VL and VM muscle force ratios and directions mainly affected M-L translation (up to $2.5 \mathrm{~mm}$ at low angles of knee flexion (below $30^{\circ}$ ), and patellar spin (up to $2^{\circ}$ ) during all flexion (Fig. 4). Variation in predicted forces was within 0.25 body weight at high angles of flexion (around 7\%).

\subsection{Patellar strain}

The constants of isotropic material law for patellar bone were identified on 200 cadaveric cubical samples with regression coefficient $r^{2}=$ 0.85 (Table 2). Mean BV/TV of cubic samples was $0.29 \pm 0.11$. Patellar bone mesh sensitivity analysis did not reveal significant difference in strain distribution prediction between meshes of 1 and $0.8 \mathrm{~mm}$. Thus, a mesh of $1 \mathrm{~mm}$ element size was used for the strain calculations.

During knee flexion, bone strain grew progressively (Fig. 6). At $30^{\circ}$, $60^{\circ}$ and $90^{\circ}$ of knee flexion, $0.01 \%, 4.3 \%$ and $19.5 \%$ of the bone volume was above $1 \%$ of octahedral shear strain respectively, while $0 \%, 0.03 \%$ and $3.9 \%$ of bone volume was above $2 \%$. With the recommended patellar cut, at $30^{\circ}, 60^{\circ}$ and $90^{\circ}$ of knee flexion $0 \%, 1.6 \%$ and $21.1 \%$ of the bone
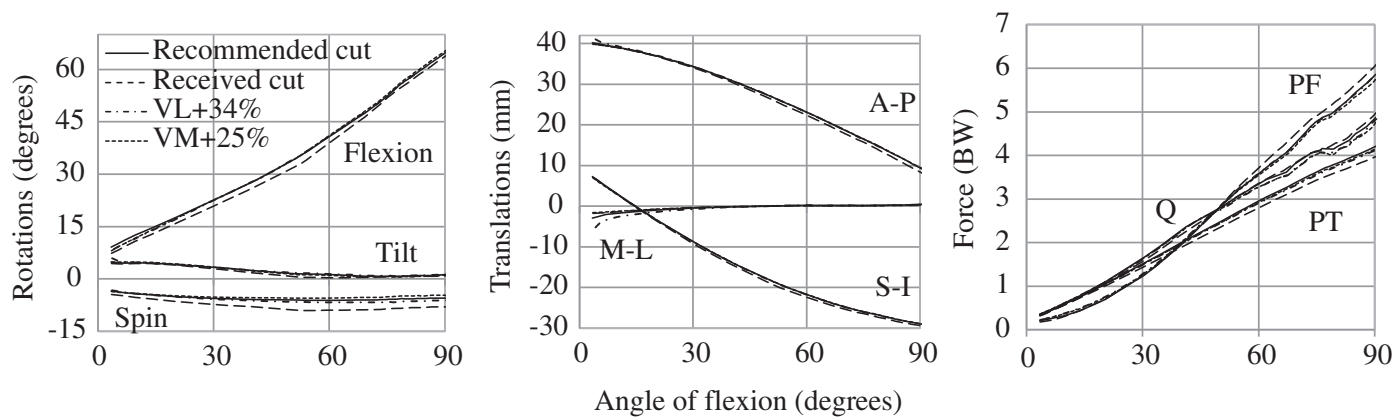

Fig. 4. Patellar kinematics and forces acting on the patella (quadriceps (Q), patellar tendon (PT) and patellofemoral force (PF)) in four simulated cases: patient received and recommended patellar cut depth, VL ratio plus $34 \%(\mathrm{VL}+34 \%)$ and VM ratio plus $25 \%$ (VM $+25 \%)$. 

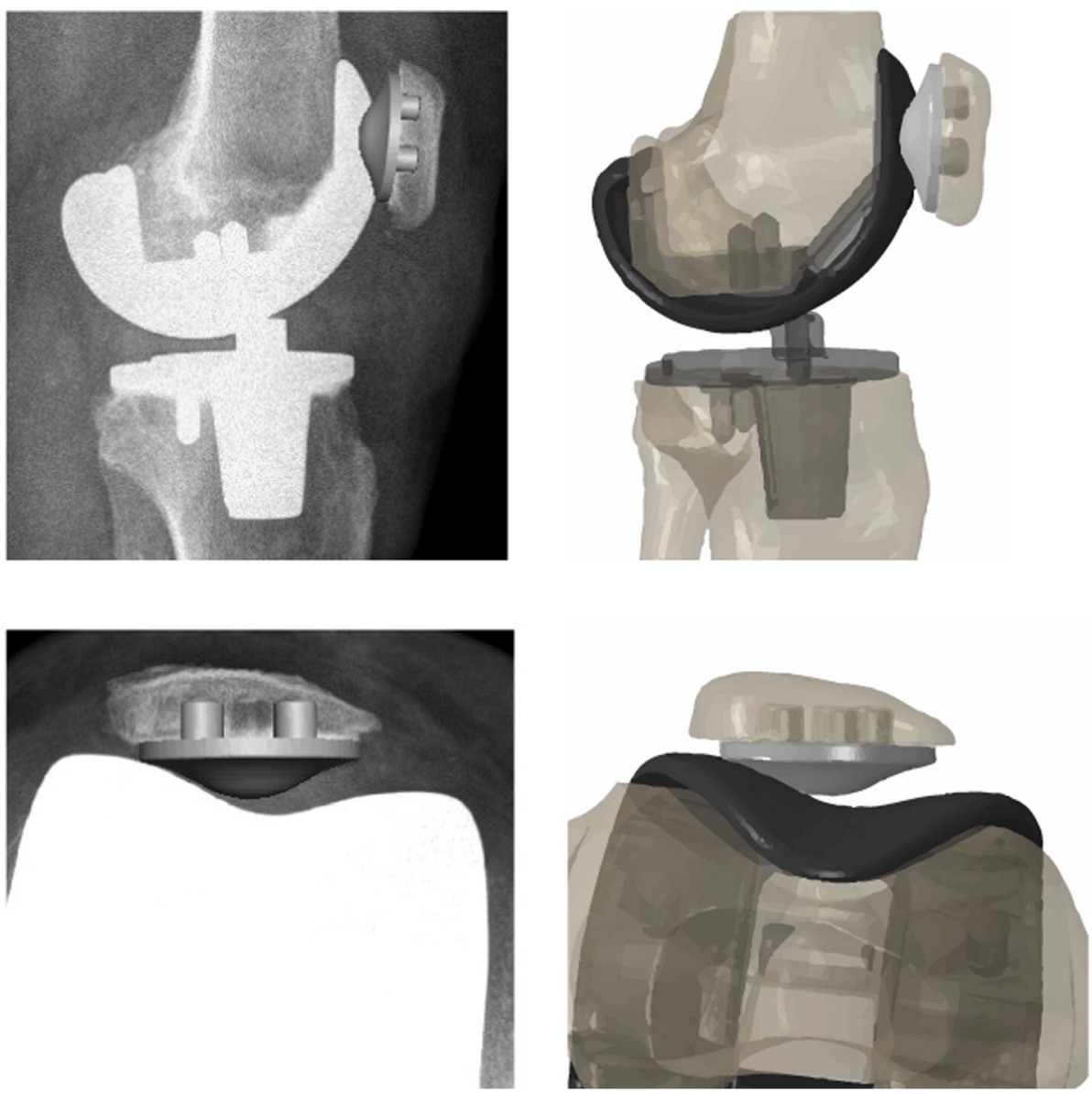

Fig. 5. Comparison of model predictions (right) with postoperative X-ray with reconstructed position of the patellar component (left) in lateral (top) and sunrise (bottom) views.

volume was above $1 \%$ of octahedral shear strain respectively, while $0 \%$, $0 \%$ and $3.8 \%$ of bone volume was above $2 \%$. Even though the patella with deeper received cut experienced higher strains (Fig. 7), two-sample Kolmogorov-Smirnov test rejected a null hypothesis that strain distributions were different in two cases.

As for sensitivity study, increase of BW by $15 \%$ (100 N) leads to almost two fold increase of the bone volume above $1 \%$ strain limit at $60^{\circ}$ of knee flexion. Results for decrease of BW were symmetrical. Change in VL and VM force ratios and directions did not change significantly volumes above $1 \%$ strain limit ( $\pm 9 \%$ of strained volume, that corresponds to $\pm 0.4 \%$ of total bone volume), but shifted it medially or laterally depending on case.

\section{Discussion}

TKA is a well-accepted surgical treatment of severe cases of osteoarthritis. Despite significant improvements in the surgical techniques during the last decades, risks of postoperative complications still exist. Especially the ones related to patella resurfacing are not fully understood. Inappropriate patellar cut is suggested to be one of the causes of complications such as patellar fracture or anterior knee pain. Estimation of patellar strain in the TKA knee can help to better understand effect of patellar cut on the possible complication development. Thus, we

Table 2

Identified constants for patellar bone material law, mean bone volume fraction ( \pm SD) among cubic samples, and coefficient of determination during regression analysis.

\begin{tabular}{lllll}
\hline$E_{0}(\mathrm{MPa})$ & $\nu_{0}$ & $k$ & $\rho$ & $r^{2}$ \\
\hline 11035.9 & 0.26 & 2.13 & $0.29 \pm 0.11$ & 0.85 \\
\hline
\end{tabular}

developed a validated numerical model to predict patient-specific patellar kinematics and strain after TKA. The model predicted that a 3-mm deeper patellar cut had a slight statistically non-significant effect on patellar kinematics and bone strain.

The model was based on patient preoperative data including imaging, preoperative implant design and BW. The kinematics predictions of the model were in good agreement with current in vivo studies (Ishimaru et al., 2014; Sharma et al., 2012). The predicted and measured quadriceps force, and the patellofemoral and patellar tendon forces were also consistent with reported values (Fitzpatrick et al., 2014b; Frohm et al., 2007; Mason et al., 2008).

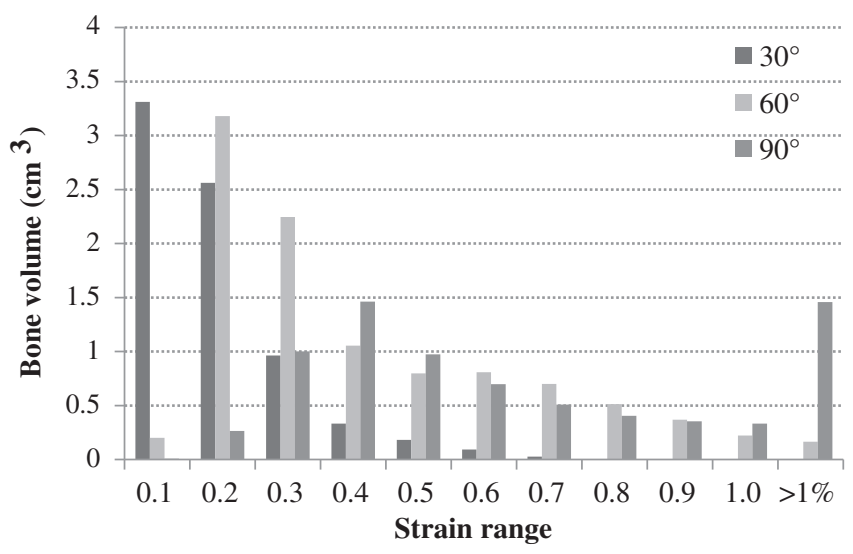

Fig. 6. Octahedral shear strain evolution in patellar bone during knee flexion. 

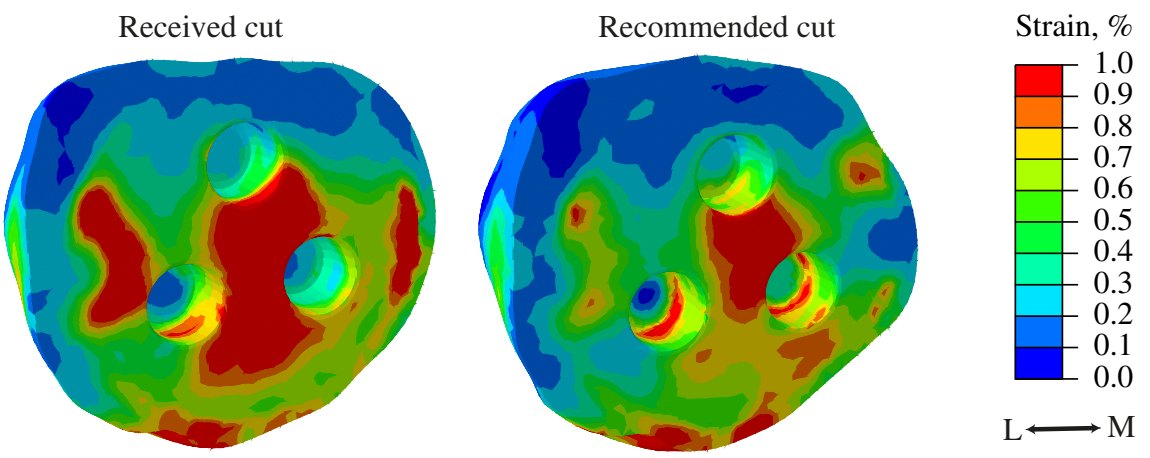

Fig. 7. Strain distribution in 2 simulated patellar cut cases at $60^{\circ}$ of knee flexion: patient postoperative cut and recommended cut.

The identified constants of the patellar bone material law were consistent with those obtained to other anatomical regions (Gross et al. 2013). The average bone volume fraction of the cubic samples corresponded to those reported in the literature (Raux et al., 1975). The predicted peak strains were compared with a similar study of (Fitzpatrick et al., 2013) and were in the same range.

The model was validated against a robotic knee simulator. The error of predicted patellar kinematics and quadriceps force during validation could be due to the simplified modeling of the patellar tendon. Even though, the kinematic predictions did not show a high sensitivity on the tendon insertion position. The error could also be related to friction and damping effects that were not included in the numerical model. In addition, position of the patella predicted with the patient-specific model was in good agreement with postoperative X-rays of the patient.

The second aim of our study was to investigate retrospectively a specific clinical case of a patient who received patellar cut deeper than recommended. We compared two cases: the deeper received cut and the recommended one. The difference between volumes above chosen strain limits was within $3 \%$ of bone volume. The statistical analysis did not show significant difference between strain distributions in bone volume for both cases. Also the patient did not have any complaints about the TKA during the 8-month follow-up period. Assuming that bone damage occurs when the exact bone volume reaches a critical strain level, our results suggest that in this case strain distribution and values of highly strained bone volume are in the acceptable range, even though deeper patellar cut increases the risk of the bone damage. The predicted strain ranges were within reported bone strain yield range. However, $1 \%$ of octahedral shear strain is probably too low to consider as a critical strain level. The strain ranges should be estimated on more patients.

Investigation of patellofemoral biomechanics on subjects by means of numerical modelling is not novel. More complex musculoskeletal subject-specific models than proposed in the current study can be found (Akbarshahi et al., 2014; Besier et al., 2005; Fitzpatrick et al., 2014a). These models require additional input data, such as joint kinematics and EMG measurements, or telemetric data. In case of retrospective study or in case of preoperative prediction of surgery outcome, such additional data can be unavailable. The strength and originality of this work were to propose a validated TKA model, which controls knee flexion through quadricep muscle activation associated with VI lengthening. VI was chosen as a reference muscle due to its central position, also VI is suggested to have a dominant role during knee extension (Zhang et al., 2003). The force within the four quadriceps and hamstring muscles was indeed calculated continuously during knee flexion, according to subject-specific data such as the anatomy of the bone and muscle, weight, knee prosthesis design or positioning. The present generic model can thus be easily adapted to account for the patient weight, the bone anatomy, the muscle anatomy, and also the prosthesis design planned for the TKA. The material law for the patellar bone was identified on cadaveric patellar bone samples. To the best of our knowledge, it is the first model that used the patellar material law obtained on patellar bone samples. And finally, the patellar strain was calculated on a TKA patient with possibility to follow-up, which allows to correlate predicted strain levels with clinical outcome.

There are several limitations of the work. The movement was not measured but simulated and was limited to the quasi-static squat and the model validation was performed only from $10^{\circ}$ to $60^{\circ}$ of flexion. We consider squat as an essential part of typical movements of daily activities (walking, chair rising, stair climbing), besides quasi-static and dynamic squat are comparable with small difference (Clement et al., 2014). The model can be extended to chair rising and gait movement with variable muscle ratios during movement if additional information (EMG, kinematics) is available. The validation was limited by the range of motion of the robotic simulator. The model validation was performed with a simplified one-muscle model, but the 7-muscle model followed similar trend, and was indirectly validated with the literature.

The muscle ratios were estimated through muscle volumes, fiber length and EMG. The error of muscle volume prediction due to interpolation was estimated to be around $4 \pm 2 \%$. The average error in PCSA calculation caused by uncertainty of fiber length is estimated to be $20 \pm$ $5 \%$. The variation in final ratios is approximately $27 \pm 4 \%$ (34\% for VL, $25 \%$ for VM and $23 \%$ for RF). The EMG measurements of VI muscle during squat movement are not known and suggested to be lower than the other muscles close to extension (Saito and Akima, 2013). The sensitivity study showed that the muscle ratio has an influence on the local distribution of the strain, but not on the total volume of strained bone. It should be taken into account if the local strain distribution is considered.

Material properties of soft tissues, such as patellofemoral ligaments, were obtained from the literature and could not be personalized. Patellofemoral ligament pre-strain corresponded to the length providing contact between patella and femur at full extension, avoiding slack ligaments. The relatively high value can be explained by the gaps induced between the patella and the femur after implantation in comparison with preoperative CT used as a reference. The collateral ligaments were not included in the model. We do not expect high influence of these ligaments on patellofemoral biomechanics, however, additional sensitivity study would be required.

The patellar bone was modeled as isotropic linear elastic material, while it is known that the patella is anisotropic due to its trabecular architecture (Toumi et al., 2006). Studies showed that modeling anisotropy could be crucial for the bone with low bone volume fraction (Hazrati Marangalou et al., 2012). Even though, the remaining after surgery anterior part of patellar bone has quite a high bone volume fraction, further investigation of the importance of anisotropy modelling on patellar bone strain predictions is needed. The identified patellar bone model was not directly validated; however, $\mu \mathrm{FE}$ modeling is currently considered as the gold standard of bone stiffness investigation in vitro, and the validity of the homogenized models based on the identified with $\mu \mathrm{FE}$ constants was shown on the other anatomical sides (Pahr et al., 2012; Pistoia et al., 2002; Wolfram et al., 2010). 
Limitations of the clinical study include consideration of a single patient. Patient follow-up was not long enough. Patellar complications may take from a few months to several years to develop, in average within the first 2 years (Chalidis et al., 2007). Further follow-up is thus needed. The study should be extended to a larger number of patients with longer follow-up to confirm the present conclusions.

\section{Conclusions}

Reduced thickness of patella after TKA surgery can be a cause of failure. With a validated patient-specific model, we predicted patellar kinematics and strain in a patient who had patellar cut deeper than recommended. By comparing for this patient, the performed cut to the recommended one, we concluded that the predicted differences in patellar kinematics and strain should be clinically negligible. We can indeed assume that the increase of the strain due to the 3-mm extra cut was acceptable in this case, since this patient had no complications, at a limited follow-up of 8 months. This validated patient-specific TKA model was used here only for a case study retrospective analysis. It showed the potential to address more complex questions, regarding surgical treatment, indication, or prosthetic design, on specific cohort of patients, and to test the hypothesis and eventually help in improvement of TKA success rate.

\section{Conflict of interest}

None of the authors have any conflict of interest.

\section{Acknowledgements}

This project was financially supported by the "Fondation de soutien à la recherche dans le domaine de l'orthopédie-traumatologie". The authors thank Elham Taghizadeh for her help in image processing, and Dr Olivier Guyen for his clinical advises.

\section{Appendix A. Supplementary data}

Supplementary data to this article can be found online at http://dx. doi.org/10.1016/j.clinbiomech.2015.11.008.

\section{References}

Akbarshahi, M., Fernandez, J.W., Schache, A.G., Pandy, M.G., 2014. Subject-specific evaluation of patellofemoral joint biomechanics during functional activity. Med. Eng. Phys. 36, 1122-1133.

Amirouche, F., Choi, K.W., Goldstein, W.M., Gonzalez, M.H., Broviak, S., 2013. Finite element analysis of resurfacing depth and obliquity on patella stress and stability in TKA. J. Arthroplast. 28, 978-984.

Anglin, C., Brimacombe, J.M., Wilson, D.R., Masri, B.A., Greidanus, N.V., Tonetti, J., Hodgson, A.J., 2008. Intraoperative vs. weightbearing patellar kinematics in total knee arthroplasty: a cadaveric study. Clin. Biomech. 23, 60-70.

Anglin, C., Fu, C., Hodgson, A.J., Helmy, N., Greidanus, N.V., Masri, B.A., 2009. Finding and defining the ideal patellar resection plane in total knee arthroplasty. J. Biomech. 42, 2307-2312.

Arami, A., Martins, N.V., Aminian, K., 2015. Locally linear neuro-fuzzy estimate of prosthetic knee angle and its validation in a robotic simulator. IEEE Sensors J. 15, 6271-6278.

Atkinson, P., Atkinson, T., Huang, C., Doane, R., 2000. A comparison of the mechanical and dimensional properties of the human medial and lateral patellofemoral ligaments. The 46th Annual Meeting of the Orthopaedic Research Society, Orlando, FL.

Besier, T.F., Gold, G.E., Beaupre, G.S., Delp, S.L., 2005. A modeling framework to estimate patellofemoral joint cartilage stress in vivo. Med. Sci. Sports Exerc. 37, 1924-1930.

Bourne, R.B., Chesworth, B.M., Davis, A.M., Mahomed, N.N., Charron, K.D., 2010. Patient satisfaction after total knee arthroplasty: who is satisfied and who is not? Clin. Orthop. Relat. Res. 468, 57-63.

Bozic, K.J., Kurtz, S.M., Lau, E., Ong, K., Chiu, V., Vail, T.P., Rubash, H.E., Berry, D.J., 2010. The epidemiology of revision total knee arthroplasty in the United States. Clin. Orthop. Relat. Res. 468, 45-51.

Carpenter, R.D., Brilhault, J., Majumdar, S., Ries, M.D., 2009. Magnetic resonance imaging of in vivo patellofemoral kinematics after total knee arthroplasty. Knee 16, 332-336.

Carter, D.R., Caler, W.E., Spengler, D.M., Frankel, V.H., 1981. Fatigue behavior of adult cortical bone: the influence of mean strain and strain range. Acta Orthop. Scand. 52, 481-490.
Chalidis, B.E., Tsiridis, E., Tragas, A.A., Stavrou, Z., Giannoudis, P.V., 2007. Management of periprosthetic patellar fractures. A systematic review of literature. Injury 38 , 714-724.

Clement, J., Hagemeister, N., Aissaoui, R., de Guise, J.A., 2014. Comparison of quasi-static and dynamic squats: a three-dimensional kinematic, kinetic and electromyographic study of the lower limbs. Gait Posture 40, 94-100.

Dionisio, V.C., Almeida, G.L., Duarte, M., Hirata, R.P., 2008. Kinematic, kinetic and EMG patterns during downward squatting. J. Electromyogr. Kinesiol. 18, 134-143.

Draper, C.E., Fredericson, M., Gold, G.E., Besier, T.F., Delp, S.L., Beaupre, G.S., Quon, A., 2012. Patients with patellofemoral pain exhibit elevated bone metabolic activity at the patellofemoral joint. J. Orthop. Res. 30, 209-213.

Fitzpatrick, C.K., Baldwin, M.A., Ali, A.A., Laz, P.J., Rullkoetter, P.J., 2011. Comparison of patellar bone strain in the natural and implanted knee during simulated deep flexion. J. Orthop. Res. 29, 232-239.

Fitzpatrick, C.K., Kim, R.H., Ali, A.A., Smoger, L.M., Rullkoetter, P.J., 2013. Effects of resection thickness on mechanics of resurfaced patellae. J. Biomech. 46, 1568-1575.

Fitzpatrick, C.K., Baldwin, M.A., Clary, C.W., Maletsky, L.P., Rullkoetter, P.J., 2014a. Evaluating knee replacement mechanics during ADL with PID-controlled dynamic finite element analysis. Comput. Methods Biomech. Biomed. Engin. 17, 360-369.

Fitzpatrick, C.K., Komistek, R.D., Rullkoetter, P.J., 2014b. Developing simulations to reproduce in vivo fluoroscopy kinematics in total knee replacement patients. J. Biomech. 47, 2398-2405.

Franceschi, J.P., Sbihi, A., 2014. 3D templating and patient-specific cutting guides (kneeplan) in total knee arthroplasty: postoperative CT-based assessment of implant positioning. Orthop. Traumatol. Surg. Res. 100, S281-S286.

Frohm, A., Halvorsen, K., Thorstensson, A., 2007. Patellar tendon load in different types of eccentric squats. Clin. Biomech. 22, 704-711.

Grood, E.S., Suntay, W.J., 1983. A joint coordinate system for the clinical description of three-dimensional motions: application to the knee. J. Biomech. Eng. 105, 136-144.

Gross, T., Pahr, D.H., Zysset, P.K., 2013. Morphology-elasticity relationships using decreasing fabric information of human trabecular bone from three major anatomical locations. Biomech. Model. Mechanobiol. 12, 793-800.

Hazrati Marangalou, J., Ito, K., van Rietbergen, B., 2012. A new approach to determine the accuracy of morphology-elasticity relationships in continuum FE analyses of human proximal femur. J. Biomech. 45, 2884-2892.

Ho, K.Y., Keyak, J.H., Powers, C.M., 2014. Comparison of patella bone strain between females with and without patellofemoral pain: a finite element analysis study J. Biomech. 47, 230-236.

Isaksson, $\mathrm{H}$., Comas, O., van Donkelaar, C.C., Mediavilla, J., Wilson, W., Huiskes, R., Ito, K. 2007. Bone regeneration during distraction osteogenesis: mechano-regulation by shear strain and fluid velocity. J. Biomech. 40, 2002-2011.

Isear Jr., J.A., Erickson, J.C., Worrell, T.W., 1997. EMG analysis of lower extremity muscle recruitment patterns during an unloaded squat. Med. Sci. Sports Exerc. 29, 532-539.

Ishimaru, M., Shiraishi, Y., Ikebe, S., Higaki, H., Hino, K., Onishi, Y., Miura, H., 2014. Threedimensional motion analysis of the patellar component in total knee arthroplasty by the image matching method using image correlations. J. Orthop. Res. 32, 619-626.

Kettenberger, U., Latypova, A., Terrier, A., Pioletti, D.P., 2015. Time course of bone screw fixation following a local delivery of zoledronate in a rat femoral model-a microfinite element analysis. J. Mech. Behav. Biomed. Mater. 45, 22-31.

Lammentausta, E., Hakulinen, M.A., Jurvelin, J.S., Nieminen, M.T., 2006. Prediction of mechanical properties of trabecular bone using quantitative MRI. Phys. Med. Biol. 51, 6187-6198.

Lie, D.T., Gloria, N., Amis, A.A., Lee, B.P., Yeo, S.J., Chou, S.M., 2005. Patellar resection during total knee arthroplasty: effect on bone strain and fracture risk. Knee Surg. Sports Traumatol. Arthrosc. 13, 203-208.

Marra, M.A., Vanheule, V., Fluit, R., Koopman, B.H., Rasmussen, J., Verdonschot, N., Andersen, M.S., 2015. A subject-specific musculoskeletal modeling framework to predict in vivo mechanics of total knee arthroplasty. J. Biomech. Eng. 137.

Mason, J.J., Leszko, F., Johnson, T., Komistek, R.D., 2008. Patellofemoral joint forces. J. Biomech. 41, 2337-2348.

Matsuda, S., Ishinishi, T., White, S.E., Whiteside, L.A., 1997. Patellofemoral joint after total knee arthroplasty. Effect on contact area and contact stress. J. Arthroplast. 12, 790-797.

Merican, A.M., Sanghavi, S., Iranpour, F., Amis, A.A., 2009. The structural properties of the lateral retinaculum and capsular complex of the knee. J. Biomech. 42, 2323-2329.

Merican, A.M., Ghosh, K.M., Baena, F.R., Deehan, D.J., Amis, A.A., 2014. Patellar thickness and lateral retinacular release affects patellofemoral kinematics in total knee arthroplasty. Knee Surg. Sports Traumatol. Arthrosc. 22, 526-533.

Morgan, E.F., Keaveny, T.M., 2001. Dependence of yield strain of human trabecular bone on anatomic site. J. Biomech. 34, 569-577.

Narici, M.V., Landoni, L., Minetti, A.E., 1992. Assessment of human knee extensor muscles stress from in vivo physiological cross-sectional area and strength measurements. Eur. J. Appl. Physiol. Occup. Physiol. 65, 438-444.

Pabinger, C., Berghold, A., Boehler, N., Labek, G., 2013. Revision rates after knee replacement. Cumulative results from worldwide clinical studies versus joint registers. Osteoarthritis Cartilage 21, 263-268.

Pahr, D.H., Dall'Ara, E., Varga, P., Zysset, P.K., 2012. HR-pOCT-based homogenised finite element models provide quantitative predictions of experimental vertebral body stiffness and strength with the same accuracy as muFE models. Comput. Methods Biomech. Biomed. Engin. 15, 711-720.

Pistoia, W., van Rietbergen, B., Lochmuller, E.M., Lill, C.A., Eckstein, F., Ruegsegger, P., 2002. Estimation of distal radius failure load with micro-finite element analysis models based on three-dimensional peripheral quantitative computed tomography images. Bone 30, 842-848.

Raux, P., Townsend, P.R., Miegel, R., Rose, R.M., Radin, E.L., 1975. Trabecular architecture of the human patella. J. Biomech. 8, 1-7. 
Saito, A., Akima, H., 2013. Knee joint angle affects EMG-force relationship in the vastus intermedius muscle. J. Electromyogr. Kinesiol. 23, 1406-1412.

Sakai, N., Luo, Z.-P., Rand, J.A., An, K.-N., 1996. Quadriceps forces and patellar motion in the anatomical model of the patellofemoral joint. Knee 3, 1-7.

Schindler, O.S., 2012. The controversy of patellar resurfacing in total knee arthroplasty: Ibisne in medio tutissimus? Knee Surg. Sports Traumatol. Arthrosc. 20, 1227-1244

Sharma, G.B., Saevarsson, S.K., Amiri, S., Montgomery, S., Ramm, H., Lichti, D.D., Lieck, R. Zachow, S., Anglin, C., 2012. Radiological method for measuring patellofemoral tracking and tibiofemoral kinematics before and after total knee replacement. Bone Joint Surg. 1, 263-271.

Staubli, H.U., Schatzmann, L., Brunner, P., Rincon, L., Nolte, L.P., 1996. Quadriceps tendon and patellar ligament: cryosectional anatomy and structural properties in young adults. Knee Surg. Sports Traumatol. Arthrosc. 4, 100-110.

Sundberg, M., Lidgren, L., W-Dahl, A., Robertsson, O., 2014. The Swedish Knee Arthroplasty Register. Annual Report (http://www.myknee.se/en/).

Takahashi, A., Sano, H., Ohnuma, M., Kashiwaba, M., Chiba, D., Kamimura, M., Sugita, T. Itoi, E., 2012. Patellar morphology and femoral component geometry influence patellofemoral contact stress in total knee arthroplasty without patellar resurfacing. Knee Surg. Sports Traumatol. Arthrosc. 20, 1787-1795.

Terrier, A., Reist, A., Vogel, A., Farron, A., 2007. Effect of supraspinatus deficiency on humerus translation and glenohumeral contact force during abduction. Clin. Biomech. 22, 645-651.

Toumi, H., Higashiyama, I., Suzuki, D., Kumai, T., Bydder, G., McGonagle, D., Emery, P., Fairclough, J. Benjamin, M. 2006. Regional variations in human patellar trabecular architecture and the structure of the proximal patellar tendon enthesis. J. Anat. 208, $47-57$.

Voigt, M., Bojsen-Moller, F., Simonsen, E.B., Dyhre-Poulsen, P., 1995. The influence of tendon Youngs modulus, dimensions and instantaneous moment arms on the efficiency of human movement. J. Biomech. 28, 281-291.

Von Keudell, A., Sodha, S., Collins, J., Minas, T., Fitz, W., Gomoll, A.H., 2014. Patient satisfac tion after primary total and unicompartmental knee arthroplasty: an age-dependent analysis. Knee $21,180-184$.

Ward, S.R., Eng, C.M., Smallwood, L.H., Lieber, R.L., 2009. Are current measurements of lower extremity muscle architecture accurate? Clin. Orthop. Relat. Res. 467, 1074-1082.

Wolfram, U., Wilke, H.J., Zysset, P.K., 2010. Valid micro finite element models of vertebral trabecular bone can be obtained using tissue properties measured with nanoindentation under wet conditions. J. Biomech. 43, 1731-1737.

Yavuz, H.U., Erdag, D., Amca, A.M., Aritan, S., 2015. Kinematic and EMG activities during front and back squat variations in maximum loads. J. Sports Sci. 1-9.

Zhang, L.-Q., Wang, G., Nuber, G.W., Press, J.M., Koh, J.L., 2003. In vivo load sharing among the quadriceps components. J. Orthop. Res. 21, 565-571.

Zysset, P.K., 2003. A review of morphology-elasticity relationships in human trabecula bone: theories and experiments. J. Biomech. 36, 1469-1485. 